\title{
Torsional instability in suspension bridges: the Tacoma Narrows Bridge case
}

\author{
Gianni ARIOLI - Filippo GAZZOLA \\ Dipartimento di Matematica - Politecnico di Milano \\ Piazza Leonardo da Vinci 32 - 20133 Milano, Italy \\ gianni.arioli@polimi.it, filippo.gazzola@polimi.it
}

\begin{abstract}
All attempts of aeroelastic explanations for the torsional instability of suspension bridges have been somehow criticised and none of them is unanimously accepted by the scientific community. We suggest a new nonlinear model for a suspension bridge and we perform numerical experiments with the parameters corresponding to the collapsed Tacoma Narrows Bridge. We show that the thresholds of instability are in line with those observed the day of the collapse. Our analysis enables us to give a new explanation for the torsional instability, only based on the nonlinear behavior of the structure.
\end{abstract}

Keywords: suspension bridges, torsional instability, Hill equation, modal analysis.

\section{Introduction}

Many suspension bridges manifested aerodynamic instability and uncontrolled oscillations leading to collapses, see e.g. [1, 22]. These accidents are due to several different causes and the focus of this paper is to analyse those due to wide torsional oscillations. Thanks to the videos available on the web [42, many people have seen the spectacular collapse of the Tacoma Narrows Bridge (TNB) occurred in 1940. The torsional oscillations were considered the main cause of the collapse [2, 40]. But the appearance of torsional oscillations is not an isolated event occurred only at the TNB: among others, we mention the collapse of the Brighton Chain Pier in 1836, the collapse of the Wheeling Suspension Bridge in 1854, the collapse of the Matukituki Suspension Footbridge in 1977. We refer to [18] for a detailed description of these collapses.

These accidents raised some fundamental questions of deep interest for both engineers and mathematicians. Due to the vortex shedding, longitudinal oscillations are to be expected in suspension bridges, but the reason of the sudden transition from longitudinal to torsional oscillations is less clear. So far, no unanimously accepted response to this question has been found. Most attempts of explanations are based on aeroelastic effects such as the frequency of the vortex shedding, parametric resonance, and flutter theory. The purpose of the present paper is to show that the origin of the torsional instability is purely structural.

Von Kármán, a member of the Board appointed for the Report [2], was convinced that the torsional motion seen on the day of the collapse was due to the vortex shedding that amplified the already present torsional oscillations and caused the center span to violent twist until the collapse, see [15, p.31]. But Scanlan [39, p.841] proved that the frequency of the torsional mode had nothing to do with the natural frequency of the shed vortices following the von Kármán vortex pattern: the calculated frequency of a vortex caused by a $68 \mathrm{~km} / \mathrm{h}$ wind is $1 \mathrm{~Hz}$, whereas the frequency of the torsional oscillations measured by Farquharson (an engineer witness of the TNB collapse, the man escaping in the video [42]) was $0.2 \mathrm{~Hz}$, see [10, p.120]. The conclusion in [10, p.122] is that the vortex trail is a 
consequence, not a primary cause of the torsional oscillation. Also Green-Unruh [20, $\S$ III] believe that vortices form independently of the motion and are not responsible for the catastrophic oscillations of the TNB. The vortex theory was later rediscussed by Larsen [27, p.247], who stated that vortices may only cause limited torsional oscillations, but cannot be held responsible for divergent large-amplitude torsional oscillations. Recently, McKenna [33] noticed that the behavior described in Larsen's paper was never observed at the Tacoma Bridge and also Green-Unruh [20] believe that the Larsen model does not adequately explain data or simulations at around $23 \mathrm{~m} / \mathrm{s}$.

Bleich [11] suggested a possible connection between the instability in suspension bridges and the flutter speed of aircraft wings; but Billah-Scanlan [10, p.122] believe that it is a great mistake to relate these two phenomena. Billah-Scanlan also claim that their own work proves that the failure of the TNB was in fact related to an aerodynamically induced condition of self-excitation in a torsional degree of freedom; but Larsen [27, p.244] believes that the work in [10] fails to connect the vortex pattern to the switch of damping from positive to negative. Moreover, McKenna [33] states that [10] is a perfectly good explanation of something that was never observed, namely small torsional oscillations, and no explanation of what really occurred, namely large vertical oscillations followed by torsional oscillations.

The parametric resonance method was adapted to a TNB model by Pittel-Yakubovich [35, 36], see also [43, Chapter VI] for the English translation and a more general setting. The conclusion on [43. p.457] claims that the most dangerous phenomenon for the stability of suspension bridges is a combination of parametric resonance. But Scanlan [39, p.841] comments these attempts by writing that Others have added to the confusion. A recent mathematics text [43], for example, seeking an application for a developed theory of parametric resonance, attempts to explain the Tacoma Narrows failure through this phenomenon. We refer to [12, 21, 24] for connections between (aerodynamic) parametric resonance, flutter theory, and self-oscillations, also applied to suspension bridges.

To conclude this quick survey of attempts for aeroelastic explanations, we mention that Scanlan [38, p.209] writes that ...the original Tacoma Narrows Bridge withstood random buffeting for some hours with relatively little harm until some fortuitous condition "broke" the bridge action over into its low antisymmetrical torsion flutter mode; the words fortuitous condition tell us that no satisfactory aeroelastic explanation is available. Due to all these controversial discussions, McKenna [32, § 2.3] writes that there is no consensus on what caused the sudden change to torsional motion, whereas Scott [40] writes that opinion on the exact cause of the Tacoma Narrows Bridge collapse is even today not unanimously shared. Summarizing, all the attempts to find a purely aeroelastic explanation of the TNB collapse fail either because the quantitative parameters do not fit the theoretical explanations or because the experiments in wind tunnels do not confirm the underlying theory.

Nowadays the attention has turned to the nonlinear behavior of structures [3, 26]. In a recent paper [4] we gave an explanation in terms of a structural instability: we considered an isolated nonlinear bridge model and we were able to show that, if the longitudinal oscillations are sufficiently small, then they are stable whereas if they are larger they can instantaneously switch to destructive torsional oscillations. The main tool used there are transfer maps (Poincaré maps), which highlight an instability when the characteristic multipliers exit the complex unit circle. The same phenomenon was later emphasized for different models using the instability for the Hill equation, see [7, 8, 9, which can also be explained by the Floquet theory. All these results were obtained by considering isolated systems, that is, by neglecting both the aerodynamic forces and the dissipation. The idea to consider an isolated system was already suggested by Irvine [23, p.176] for a suspension bridge model similar to the one considered in [4]: Irvine ignores damping of both structural and aerodynamic origin, his purpose being to simplify as much as possible the model by maintaining its essence, that is, the conceptual design of bridges. And since our purpose is precisely to highlight the role of the structure on the stability, we follow this suggestion: in the conclusions of the present paper we mention how the aerodynamic effects combine with the structural effects.

In this paper we improve the results in [4] by introducing a more precise model which also takes 
into account the nonlinear restoring action of the cables+hangers system on the deck; the model is described in detail in Section 2 and is the nonlinear version of a linear model that we introduced in [5]. In Section 3 we define the nonlinear longitudinal modes, that is, the periodic purely longitudinal motions of the deck which may be unstable and create torsional motions. In Sections 4.1 and 4.2 we describe the theoretical framework that we use to study the stability of the longitudinal modes and we prove that they are stable for small energies. Finally, we validate our theory by performing numerical simulations of the model with the parameters of the collapsed TNB. We study the existence and the behavior of approximate longitudinal modes and we compute their instability thresholds. Our numerical results confirm that the longitudinal oscillations with 8 or 9 nodes and amplitudes of about $4 \mathrm{~m}$, namely the oscillations observed the day of the collapse, are prone to generate torsional oscillations, see Section 4.3. The main conclusions and our explanation of the TNB collapse are collected in Section 5 .

\section{The mathematical model}

\subsection{Description of the structure}

Throughout this paper the variable $x$ indicates the position along the deck and we denote the space and time derivatives as follows:

$$
w=w(x) \Rightarrow w^{\prime}=\frac{d w}{d x}, \quad w=w(t) \Rightarrow \dot{w}=\frac{d w}{d t}, \quad w=w(x, t) \Rightarrow\left\{\begin{array}{l}
w_{x}=\frac{\partial w}{\partial x} \\
w_{t}=\frac{\partial w}{\partial t}
\end{array}\right.
$$

and similarly for higher order derivatives. The physical constants are listed in Section 4.4.

In a suspension bridge four towers sustain two cables that, in turn, sustain the hangers. At their lower endpoint the hangers are linked to the deck and sustain it from above. The hangers are hooked to the cables and the deck is hooked to the hangers. In this section we quickly revisit the bridge model that we recently introduced in [5]: the displacements involved were assumed to be small, which justifies the asymptotic expansion of the energies. Here we refine this model by considering more terms: this raises several nonlinearities in the Euler-Lagrange equations. Moreover, we neglect the flexibility of the hangers: this will be justified below. As usual in engineering literature, we assume that positive displacements of the deck and the cables are oriented downwards and the origin is at the level of the deck at rest.

We assume that the deck has length $L$ and width $2 \ell$ with $2 \ell \ll L$; we model it as a degenerate plate consisting of a beam representing the midline and cross sections which can rotate around the beam. The beam contains the barycenters of the cross sections and we denote its position with $y=y(x, t)$. The angle of rotation of the cross sections with respect to the horizontal position is denoted by $\theta=\theta(x, t)$. Then the positions of the free edges of the deck are given by $y \pm \ell \sin \theta \approx y \pm \ell \theta$ since we aim to study what happens in a small torsional regime. We emphasise that our results do not aim to describe the behavior of the bridge when the torsional angle becomes large; instead, we explain how a small torsional angle can suddenly increase, that is, our purpose is to describe the mechanism that triggers torsional oscillations.

Since the spacing between hangers is small relative to the span, the hangers can be considered as a continuous membrane connecting the cables and the deck. We denote by $-s(x)$ the position of the cables at rest, $-s_{0}<0$ being the level of the left and right endpoints of the cables $\left(s_{0}\right.$ is the height of the towers); $L$ is the distance between the towers. If a beam of length $L$ and linear density of mass $M$ is hanged to a cable whose linear density of mass is $m$, and if $g$ denotes the gravitational constant, then the cable at rest is subject to a downwards vertical force density given by $\left(M+m \sqrt{1+s^{\prime}(x)^{2}}\right) g$. Each free side of the deck is connected with hangers to a cable so that each cable sustains the weight 
of half deck; then, we obtain the following boundary value problem for $s(x)$ :

$$
\left\{\begin{array}{l}
H_{0} s^{\prime \prime}(x)=\left(\frac{M}{2}+m \sqrt{1+s^{\prime}(x)^{2}}\right) g, \\
s(0)=s(L)=s_{0} .
\end{array}\right.
$$

It is not difficult to show that (1) admits a unique solution which, moreover, is symmetric with respect to $x=L / 2$, see [5, Proposition 1] for the details. In order to simplify notations we set

$$
\xi(x):=\sqrt{1+s^{\prime}(x)^{2}},
$$

so that, $\xi(x)$ represents the local length of the cable at rest. When the deck is mounted, the hangers are in tension and reach the length $s(x)$, where $s$ is the solution of (1); if no additional load acts on the system, the equilibrium position of the deck is horizontal and the position of the cables at equilibrium (deck mounted) is $-s(x)$ for $x \in(0, L)$.

The two sustaining cables are labeled with $i=1,2$. If $p_{i}=p_{i}(x, t)$ denotes the displacement of the cable $i$ with respect to the rest position, then $p_{i}(x, t)-s(x)$ denotes the actual position of the cable.

The flexibility of the hangers has a significant effect on the frequencies of the higher modes when the deck is very stiff, see [31]. However, as far as lower modes and weakly stiffened bridges are involved, the elastic deformation of the hangers may be neglected, see again the results by Luco-Turmo [31]. Since we aim to model a very flexible deck as the TNB, we assume that the hangers are rigid, their action merely being to connect the deck to the cables. This assumption is justified by the fact that the action of the cables is the main cause of the nonlinearity of the restoring force, see Bartoli-Spinelli $[\underline{6}$, p.180]. Moreover, the nonlinear contribution of the hangers is mainly due to their slackening but, as reported in [2], the slackening of the hangers occurred at the TNB only after that the large torsional oscillations appeared. Our purpose is to understand how negligible torsional oscillations of the deck suddenly become dangerous ones, that is, to describe what happens before the slackening starts. This is a further reason to consider rigid hangers. If we assume that the hangers have a fixed length, then $p_{1}=y+\ell \theta$ and $p_{2}=y-\ell \theta$ : for our convenience, in the next subsection we maintain the notation with the $p_{i}$ 's while we replace them in the Euler-Lagrange equations (6)-(7).

\subsection{Kinetic and potential energies of the structure}

The kinetic energy of the deck is the sum of the kinetic energy of the barycenter of the cross section and of the kinetic energy of the torsional angle. Then the total kinetic energy of the bridge is

$$
E_{\mathrm{kin}}=\frac{M}{2} \int_{0}^{L}\left(\frac{\ell^{2} \theta_{t}^{2}}{3}+y_{t}^{2}\right) d x+\frac{m}{2} \int_{0}^{L}\left[\left(p_{1}\right)_{t}^{2}+\left(p_{2}\right)_{t}^{2}\right] \xi(x) d x .
$$

The gravitational energies of the deck and the cables are, respectively, given by

$$
-M g \int_{0}^{L} y d x \quad \text { and } \quad-m g \int_{0}^{L}\left(p_{1}+p_{2}\right) \xi(x) d x .
$$

The elastic energy of the deck is composed by the bending energy of the beam and the torsional energy:

$$
E_{\mathrm{el}}=\frac{E I}{2} \int_{0}^{L} y_{x x}^{2} d x+\frac{G K}{2} \int_{0}^{L} \theta_{x}^{2} d x
$$

The tension of each cable consists of two parts. The first part is the tension at rest $H(x)=H_{0} \xi(x)$. The amount of energy needed to deform each cable at rest under the tension $H(x)$ in the infinitesimal interval $[x, x+d x]$ from the original position $-s(x)$ to $-s(x)+p_{i}(x, t)(i=1,2)$ is the variation of length times the tension, that is

$$
E_{c}(x) d x=H_{0} \xi(x)\left(\sqrt{1+\left[s^{\prime}(x)-\left(p_{i}\right)_{x}(x, t)\right]^{2}}-\sqrt{1+s^{\prime}(x)^{2}}\right) d x .
$$


Hence, using the asymptotic expansion

$$
\sqrt{1+\left[s^{\prime}-\left(p_{i}\right)_{x}\right]^{2}}-\sqrt{1+\left(s^{\prime}\right)^{2}}=-\frac{s^{\prime}\left(p_{i}\right)_{x}}{\xi}+\frac{\left(p_{i}\right)_{x}^{2}}{2 \xi^{3}}+\frac{s^{\prime}\left(p_{i}\right)_{x}^{3}}{2 \xi^{5}}+o\left(\left(p_{i}\right)_{x}^{3}\right)
$$

and neglecting the higher order terms, the energy necessary to deform the whole cable is

$$
E_{c}\left(p_{i}\right)=\int_{0}^{L} E_{c}(x) d x \approx H_{0} \int_{0}^{L}\left(-s^{\prime}\left(p_{i}\right)_{x}+\frac{\left(p_{i}\right)_{x}^{2}}{2 \xi^{2}}+\frac{s^{\prime}\left(p_{i}\right)_{x}^{3}}{2 \xi^{4}}\right) d x .
$$

The second part of the tension of each cable is the additional tension

$$
\frac{A E}{L_{c}} \Gamma\left(p_{i}\right)
$$

due to the increment of length $\Gamma\left(p_{i}\right)$ of the cable $(i=1,2)$. This increment requires the energy

$$
E_{t c}\left(p_{i}\right)=\frac{A E}{2 L_{c}} \Gamma\left(p_{i}\right)^{2}
$$

In view of the asymptotic expansion (2), we can approximate the increment $\Gamma\left(p_{i}\right)$ of the length of the cable (due to its displacement $p_{i}$ ) by

$$
\Gamma\left(p_{i}\right):=\int_{0}^{L} \sqrt{1+\left[s^{\prime}-\left(p_{i}\right)_{x}\right]^{2}} d x-L_{c} \approx \int_{0}^{L}\left(\frac{-s^{\prime}\left(p_{i}\right)_{x}}{\xi}+\frac{\left(p_{i}\right)_{x}^{2}}{2 \xi^{3}}\right) d x .
$$

A third order expansion then gives

$$
\Gamma\left(p_{i}\right)^{2}=\left(\int_{0}^{L} \frac{s^{\prime}\left(p_{i}\right)_{x}}{\xi}\right)^{2}-\left(\int_{0}^{L} \frac{s^{\prime}\left(p_{i}\right)_{x}}{\xi}\right)\left(\int_{0}^{L} \frac{\left(p_{i}\right)_{x}^{2}}{\xi^{3}}\right)+o\left(\left(p_{i}\right)_{x}^{3}\right) .
$$

Therefore, we approximate (4) with

$$
E_{t c}\left(p_{i}\right)=\frac{A E}{2 L_{c}}\left(\int_{0}^{L} \frac{s^{\prime}\left(p_{i}\right)_{x}}{\xi}\right)^{2}-\frac{A E}{2 L_{c}}\left(\int_{0}^{L} \frac{s^{\prime}\left(p_{i}\right)_{x}}{\xi}\right)\left(\int_{0}^{L} \frac{\left(p_{i}\right)_{x}^{2}}{\xi^{3}}\right) .
$$

\subsection{The Euler-Lagrange equations}

Some integration by parts show that the variation of the energy $E_{c}\left(p_{i}\right)$ in $(3)$ is given by

$$
d E_{c}\left(p_{i}\right) z=H_{0} \int_{0}^{L}\left(s^{\prime}-\frac{\left(p_{i}\right)_{x}}{\xi^{2}}-\frac{3 s^{\prime}\left(p_{i}\right)_{x}^{2}}{2 \xi^{4}}\right)_{x} z d x .
$$

For the variation of the energy $E_{t c}\left(p_{i}\right)$ in (5), we neglect terms of order larger than 2 , and by integrating by parts we obtain

$$
d E_{t c}\left(p_{i}\right) z=\frac{A E}{2 L_{c}}\left(\int_{0}^{L} \frac{\left(p_{i}\right)_{x}^{2}}{\xi^{3}}\right) \int_{0}^{L} \frac{s^{\prime \prime}}{\xi^{3}} z d x+\frac{A E}{L_{c}}\left(\int_{0}^{L} \frac{s^{\prime \prime} p_{i}}{\xi^{3}}\right) \int_{0}^{L}\left(\frac{s^{\prime}}{\xi}-\frac{\left(p_{i}\right)_{x}}{\xi^{3}}\right)_{x} z d x .
$$

By recalling that $p_{1}=y+\ell \theta$ and $p_{2}=y-\ell \theta$, the first variation of the sum of all the energies involved yields the following Euler-Lagrange equations:

$$
\begin{aligned}
(M+2 m \xi) y_{t t}= & -E I y_{x x x x}+H_{0}\left(\frac{2 y_{x}}{\xi^{2}}+3 \frac{s^{\prime}\left(y_{x}^{2}+\ell^{2} \theta_{x}^{2}\right)}{\xi^{4}}\right)_{x}-\frac{A E}{L_{c}}\left[\int_{0}^{L} \frac{y_{x}^{2}+\ell^{2} \theta_{x}^{2}}{\xi^{3}}\right] \frac{s^{\prime \prime}}{\xi^{3}} \\
& -\frac{2 A E}{L_{c}}\left[\int_{0}^{L} \frac{s^{\prime \prime} y}{\xi^{3}}\right]\left(\frac{s^{\prime}}{\xi}-\frac{y_{x}}{\xi^{3}}\right)_{x}+\frac{2 A E \ell^{2}}{L_{c}}\left[\int_{0}^{L} \frac{s^{\prime \prime} \theta}{\xi^{3}}\right]\left(\frac{\theta_{x}}{\xi^{3}}\right)_{x}, \\
\left(\frac{M}{3}+2 m \xi\right) \theta_{t t}= & \frac{G K}{\ell^{2}} \theta_{x x}+2 H_{0}\left(\frac{\theta_{x}}{\xi^{2}}+3 \frac{s^{\prime} y_{x} \theta_{x}}{\xi^{4}}\right)_{x}-\frac{2 A E}{L_{c}}\left[\int_{0}^{L} \frac{y_{x} \theta_{x}}{\xi^{3}}\right] \frac{s^{\prime \prime}}{\xi^{3}} \\
& -\frac{2 A E}{L_{c}}\left[\int_{0}^{L} \frac{s^{\prime \prime} \theta}{\xi^{3}}\right]\left(\frac{s^{\prime}}{\xi}-\frac{y_{x}}{\xi^{3}}\right)_{x}+\frac{2 A E}{L_{c}}\left[\int_{0}^{L} \frac{s^{\prime \prime} y}{\xi^{3}}\right]\left(\frac{\theta_{x}}{\xi^{3}}\right)_{x} .
\end{aligned}
$$


This is the system describing the dynamics of a suspension bridge that we will consider throughout the paper. Since the degenerate plate is hinged between the two towers and the cross sections between the towers cannot rotate, the boundary conditions to be associated to (6)-(7) read

$$
y(0, t)=y(L, t)=y_{x x}(0, t)=y_{x x}(L, t)=\theta(0, t)=\theta(L, t)=0 \quad \forall t \geq 0 .
$$

For our investigation of (6)-(7) (both theoretical and numerical) we first set $\theta \equiv 0$ in (6) to obtain

$$
(M+2 m \xi) y_{t t}+E I y_{x x x x}=H_{0}\left(\frac{2 y_{x}}{\xi^{2}}+\frac{3 s^{\prime} y_{x}^{2}}{\xi^{4}}\right)_{x}-\frac{A E}{L_{c}}\left[\int_{0}^{L} \frac{y_{x}^{2}}{\xi^{3}}\right] \frac{s^{\prime \prime}}{\xi^{3}}-\frac{2 A E}{L_{c}}\left[\int_{0}^{L} \frac{s^{\prime \prime} y}{\xi^{3}}\right]\left(\frac{s^{\prime}}{\xi}-\frac{y_{x}}{\xi^{3}}\right)_{x}
$$

and we find periodic solutions $y$ of different amplitude and number of zeros. Then, for each solution $y$ of (9) we consider (7) as a linear equation in $\theta$ with periodic coefficients and we study the stability of the trivial solution of (7).

Our characterization of torsional stability consists in taking a periodic solution $y$ of $(9)$ and plugging it into (7); we say that $y$ is torsionally stable if the trivial solution of the so obtained equation (see (19) below) is stable. This is basically the characterization suggested by Dickey [16] for a couple of oscillators related to a nonlinear string: it is also called linear stability in literature [19, Definition 2.3]. An apparently stronger form of stability, without linearization and substitution of $y$, is the well-known Lyapunov characterization. For a simpler system of ODEs Ghisi-Gobbino [19] were able to prove that these two characterizations are equivalent.

\section{Nonlinear longitudinal modes}

The milestone paper by Rabinowitz [37] started the systematic study of the existence of periodic solutions for the nonlinear string equation

$$
u_{t t}-u_{x x}+f(x, u)=0 \quad(x, t) \in(0, \pi) \times \mathbb{R}_{+}, \quad u(0, t)=u(\pi, t)=0 \quad t \in \mathbb{R}_{+} .
$$

Prior to [37, only perturbation techniques were available and the nonlinearity was assumed to be small in a suitable sense. Rabinowitz proved that (10) admits periodic solutions. In fact, there exist infinitely many periodic solutions of (10), having "almost" any possible period $T>0$ : quite sophisticated tools are necessary to obtain a full description of the set of periodic solutions, see e.g. [17] and references therein. Periodic solutions were also found for the related nonlinear beam equation

$$
\begin{cases}u_{t t}+u_{x x x x}+f(x, u)=0 & (x, t) \in(0, \pi) \times \mathbb{R}_{+}, \\ u(0, t)=u(\pi, t)=u_{x x}(0, t)=u_{x x}(\pi, t)=0 & t \in \mathbb{R}_{+},\end{cases}
$$

see [28, 29, 30]. Also nonlocal (and nonlinear) variants of (10) have been considered. In 1876 Kirchhoff 25] introduced the following nonlinear vibrating string equation

$$
u_{t t}-\left(a+b \int_{0}^{\pi} u_{x}^{2}\right) u_{x x}=0 \quad(x, t) \in(0, \pi) \times \mathbb{R}_{+}, \quad u(0, t)=u(\pi, t)=0 \quad t \in \mathbb{R}_{+}
$$

where $a, b>0$. Note that 10 with $f(x, u)=a u+b u^{3}$ is quite similar to 12 and, in some respect, 12 appears more complicated since first order derivatives are involved. Nevertheless, the modal analysis of 12 is much simpler since it behaves as a "hidden" linear problem. Exploiting this peculiarity, Cazenave-Weissler 13 proved that $(12)$ has infinitely many periodic solutions.

If we take $\theta(x, 0)=\theta_{t}(x, 0)=0$ as initial data in (6)-(7)-(8), then $\theta(x, t) \equiv 0$, whereas $y$ solves the nonlinear nonlocal equation (9). A careful look at (9) shows that its structure, its nonlinearities, and its nonlocal terms are of the same kind as those appearing in (10), (11), and (12). It is therefore reasonable to conjecture that (9) admits infinitely many periodic-in-time solutions. 
In order to find such solutions, or at least a reliable approximation of them, we proceed numerically. The first step is to look for small periodic solutions of $(9)$ close to those of the linearized problem

$$
(M+2 m \xi) y_{t t}+E I y_{x x x x}=2 H_{0}\left(\frac{y_{x}}{\xi^{2}}\right)_{x}-\frac{2 A E}{L_{c}}\left[\int_{0}^{L} \frac{s^{\prime \prime} y}{\xi^{3}}\right] \frac{s^{\prime \prime}}{\xi^{3}} .
$$

Let us analyze (13) and see what has to be expected from the numerical procedure. Periodic solutions of (13) may be obtained by separating variables. Consider the linear operator $\mathcal{L}$ defined by

$$
\mathcal{L} u:=E I u^{\prime \prime \prime \prime}-2 H_{0}\left(\frac{u^{\prime}}{\xi^{2}}\right)^{\prime}+\frac{2 A E}{L_{c}}\left[\int_{0}^{L} \frac{s^{\prime \prime} u}{\xi^{3}}\right] \frac{s^{\prime \prime}}{\xi^{3}} \quad \forall u \in C^{4}[0, L] .
$$

The definition of $\mathcal{L}$ may be continuously extended to the Sobolev space $H^{2} \cap H_{0}^{1}(0, L)$ : the operator $\mathcal{L}$ maps this space into its dual space $H^{\prime}$ and is implicitly defined by

$$
\langle\mathcal{L} u, v\rangle=E I \int_{0}^{L} u^{\prime \prime} v^{\prime \prime}+2 H_{0} \int_{0}^{L} \frac{u^{\prime} v^{\prime}}{\xi^{2}}+\frac{2 A E}{L_{c}}\left[\int_{0}^{L} \frac{s^{\prime \prime} u}{\xi^{3}}\right]\left[\int_{0}^{L} \frac{s^{\prime \prime} v}{\xi^{3}}\right] \quad \forall u, v \in H^{2} \cap H_{0}^{1}(0, L)
$$

where $\langle\cdot, \cdot\rangle$ denotes the duality pairing between $H^{2} \cap H_{0}^{1}(0, L)$ and $H^{\prime}$. The bilinear form in (14) is symmetric and coercive. Therefore, the operator $\mathcal{L}$ is self-adjoint and the eigenvalue problem

$$
\mathcal{L} u=\lambda(M+2 m \xi) u
$$

admits an unbounded sequence $\left\{\lambda_{k}\right\}$ of positive eigenvalues whose corresponding eigenfunctions form a complete system in $H^{2} \cap H_{0}^{1}(0, L)$.

If the cables were in the limit horizontal position, that is $s^{\prime}=s^{\prime \prime}=0$ and $\xi=1$, then the eigenfunctions of 15 would be $\sin \left(\frac{k \pi}{L} x\right)$ for all integer $k \geq 1$ with corresponding eigenvalues given by

$$
\lambda_{k}=\frac{E I k^{4} \pi^{4}+2 H_{0} k^{2} \pi^{2} L^{2}}{(M+2 m) L^{4}} .
$$

As we could verify numerically, when the shape of the cable satisfies (1), the eigenfunctions of (15) remain close to $\sin \left(\frac{k \pi}{L} x\right)$ with $k-1$ zeros in $(0, L)$.

Denote by $\varphi_{\lambda}$ an eigenfunction of (15) relative to the eigenvalue $\lambda$, then for any $A, B \in \mathbb{R}$ the function

$$
u(x, t)=\varphi_{\lambda}(x)(A \sin (\sqrt{\lambda} t)+B \cos (\sqrt{\lambda} t))
$$

is a periodic solution of 13 .

We numerically find approximate periodic solutions for (9) in two steps. First, we transform the PDE into a system of ODEs by discretising in space via Fourier polynomials and we build a Mathematica program that solves the corresponding initial value problems. Then we look for points in the (finite dimensional) phase space that belong to a periodic orbit.

More precisely, given the boundary conditions (8), we look for an approximate solution of (9) that can be represented by a trigonometric polynomial

$$
y(x, t)=\sum_{k=1}^{n} y_{k}(t) \sin \left(\frac{k \pi x}{L}\right) .
$$

To achieve this goal, we plug (17) into $(9)$ and we project it onto the span of $\{\sin (\pi x / L), \ldots, \sin (n \pi x / L)\}$ : in particular, we choose initial conditions

$$
y(x, 0)=\sum_{k=1}^{n} y_{0 k} \sin \left(\frac{k \pi x}{L}\right), \quad y_{t}(x, 0)=\sum_{k=1}^{n} y_{1 k} \sin \left(\frac{k \pi x}{L}\right)
$$


for some $Y_{0}=\left\{y_{0 k}\right\}_{k=1, \ldots, n} \in \mathbb{R}^{n}$ and $Y_{1}=\left\{y_{1 k}\right\}_{k=1, \ldots, n} \in \mathbb{R}^{n}$. The dimension $n$ is chosen in such a way that the result of the numerical integration does not change significantly when $n$ is increased. For the computation of the first 6 periodic solutions we take $n=10$, while for the computation of the next 4 periodic solutions we take $n=16$.

Let $Y(t)=\left\{y_{k}(t)\right\}_{k=1, \ldots, n} \in \mathbb{R}^{n}$; we obtain a system of $n$ nonlinear ODEs in the form

$$
\ddot{Y}(t)=G(Y(t)) \quad\left(G: \mathbb{R}^{n} \rightarrow \mathbb{R}^{n}\right)
$$

with initial conditions $Y(0)=Y_{0}$ and $\dot{Y}(0)=Y_{1}$. We use Newton's algorithm to find initial vectors $Y_{0}$ and $Y_{1}$ that lead to periodic solutions of (18). For any $T>0$ we define the transfer map

$$
\Phi_{T}: \mathbb{R}^{2 n} \rightarrow \mathbb{R}^{2 n}, \quad \Phi_{T}\left(Y_{0}, Y_{1}\right)=(Y(T), \dot{Y}(T)),
$$

where $Y(t)$ is the solution of (18) with initial conditions $\left(Y_{0}, Y_{1}\right)$, and we look for fixed points of $\Phi_{T}$ by iterating the transformation

$$
N\left(Y_{0}, Y_{1}\right):=\left(Y_{0}, Y_{1}\right)-\left[J \Phi_{T}\left(Y_{0}, Y_{1}\right)-I\right]^{-1}\left(\Phi_{T}\left(Y_{0}, Y_{1}\right)-\left(Y_{0}, Y_{1}\right)\right)
$$

where $J \Phi_{T}$ is the Jacobian matrix of $\Phi_{T}$ and $I$ is the identity $2 n \times 2 n$ matrix. As initial point for the Newton's algorithm we choose $Y_{0}=\alpha e_{k}$ and $Y_{1}=0$, where $\alpha$ is some positive number, $k \in\{1, \ldots, n\}$ and $\left\{e_{1}, \ldots, e_{n}\right\}$ is the canonical basis in $\mathbb{R}^{n}$. We observe that, if $\alpha$ is sufficiently small, there exists $T>0$ such that $\Phi_{T}\left(Y_{0}, Y_{1}\right) \simeq\left(Y_{0}, Y_{1}\right)$ : for such $T$, Newton's algorithm converges rapidly. In Table 1 we quote the period $T$ of each mode obtained for $\alpha \rightarrow 0$.

\begin{tabular}{|c|c|c|c|c|c|c|c|c|c|c|}
\hline Mode & 1 & 2 & 3 & 4 & 5 & 6 & 7 & 8 & 9 & 10 \\
\hline Period $(s)$ & 10.95 & 7.67 & 5.42 & 3.75 & 2.9 & 2.41 & 2.02 & 1.72 & 1.5 & 1.32 \\
\hline
\end{tabular}

Table 1: Period of the longitudinal mode on each branch for energies close to 0 .

Figure 1 represents the graph of the solution obtained by this algorithm for $k=8$ at time $t=0$; as expected, it resembles a multiple of $\sin \left(\frac{8 \pi}{L} x\right)$ (with $L=853.44 \mathrm{~m}$, see Section 4.4). Since this function has small $L^{\infty}$-norm, we expect it to be an approximate periodic solution of the linear equation $(13)$.

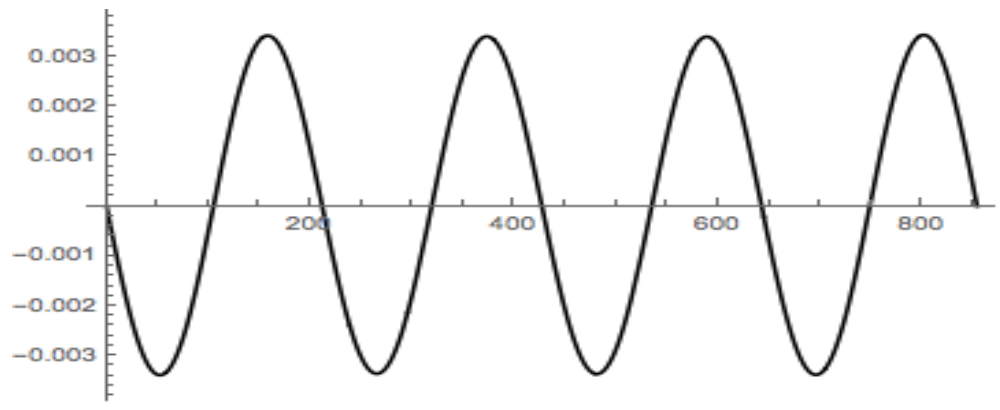

Figure 1: $y(x, 0)$ for the solution on the 8 th branch at $T=1.72$ (small energy).

This small periodic solution of the nonlinear system $(18)$ is an example of what we expect to observe when a still bridge is slightly perturbed by some external forcing, typically the wind. If more energy is inserted into the structure, the oscillation becomes wider. Since the insertion of energy is gradual, it seems natural to look for other solutions, of larger energy and $L^{\infty}$-norm, by continuity. To do so, we slightly increase $T$ and use again Newton's algorithm: it turns out that, on each branch, the period is an increasing function of the energy and, if the increment in $T$ is sufficiently small, Newton's 
algorithm keeps converging rapidly, and the resulting solution of 18 has larger energy and $L^{\infty}$-norm. By increasing $T$ we build the branch of the $k$-th nonlinear modes of $(18)$. This procedure cannot be extended indefinitely; at some point the convergence of Newton's algorithm starts deteriorating and smaller increments in $T$ must be taken in order to keep following the branch of solutions. We stop the procedure when the acceptable increments become too small to be feasible. In this way we obtain all the branches of the $k$-th nonlinear longitudinal modes for $k=1, \ldots, 10$.

Definition 1. We call a periodic solution of (18) obtained with this algorithm a $k$-th nonlinear longitudinal mode of period $T$.

We observed that the nonlinear longitudinal modes on the odd branches are symmetric with respect to the center of the bridge, see e.g. Figure 2 where the solution on the 7 th branch at $T=2.18$ is displayed. On the other hand, the modes on even branches have no symmetries, see e.g. Figure 3 where the solution on the 8 th branch at $T=1.86$ is displayed.

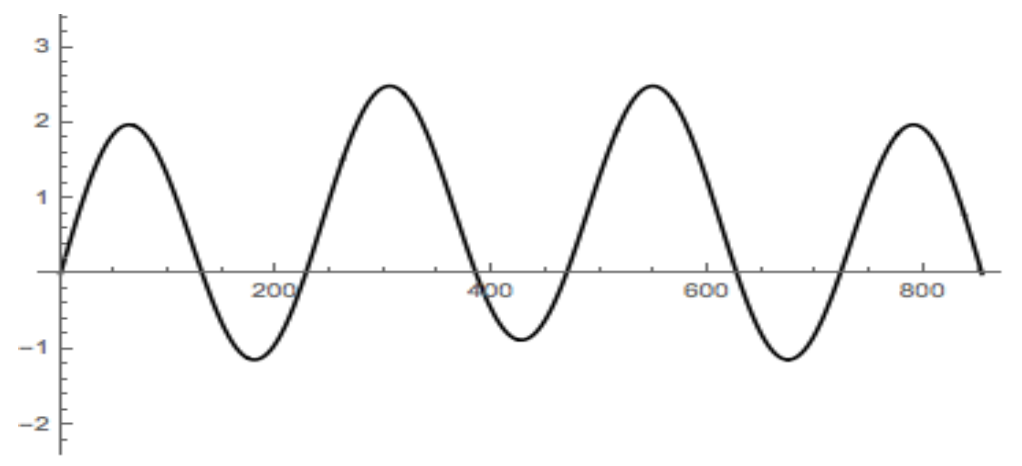

Figure 2: $y(x, 0)$ for the solution on the 7 th branch at $T=2.18$ (critical threshold of instability).

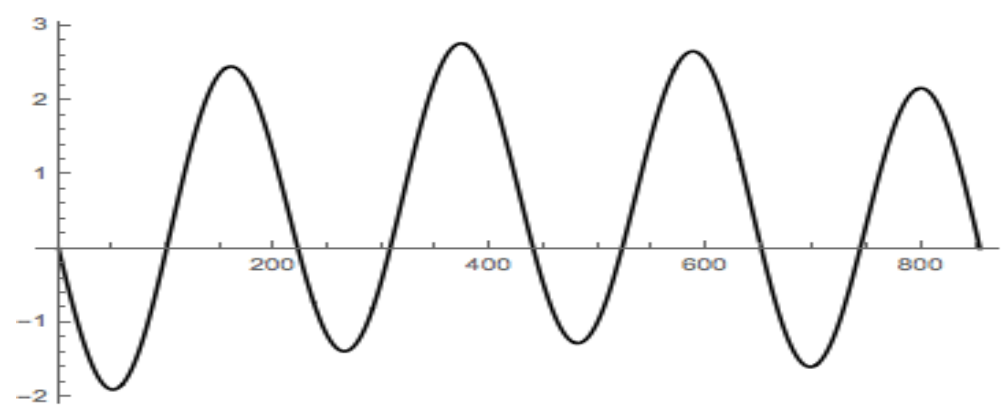

Figure 3: $y(x, 0)$ for the solution on the 8 th branch at $T=1.86$ (critical threshold of instability).

We remark that the $k$-th Fourier component of a $k$-th nonlinear longitudinal mode is much larger than the other ones, see e.g. Figure 4 where the evolution in time of the first 12 Fourier components for the solution obtained with $k=8$ with $T=1.86$ is displayed.

In all our experiments we also observed that the first Fourier component of the periodic solution (namely the function $y_{1}(t)$ in $(17)$ ) is negative for all times, see e.g. the first plot in Figure 4 . This means that the deck is bent upwards during periodic motions. In fact, our results show that, when the periodic motion starts, the cables undergo an additional tension due to the nonlocal term and therefore they pull the deck upwards. 


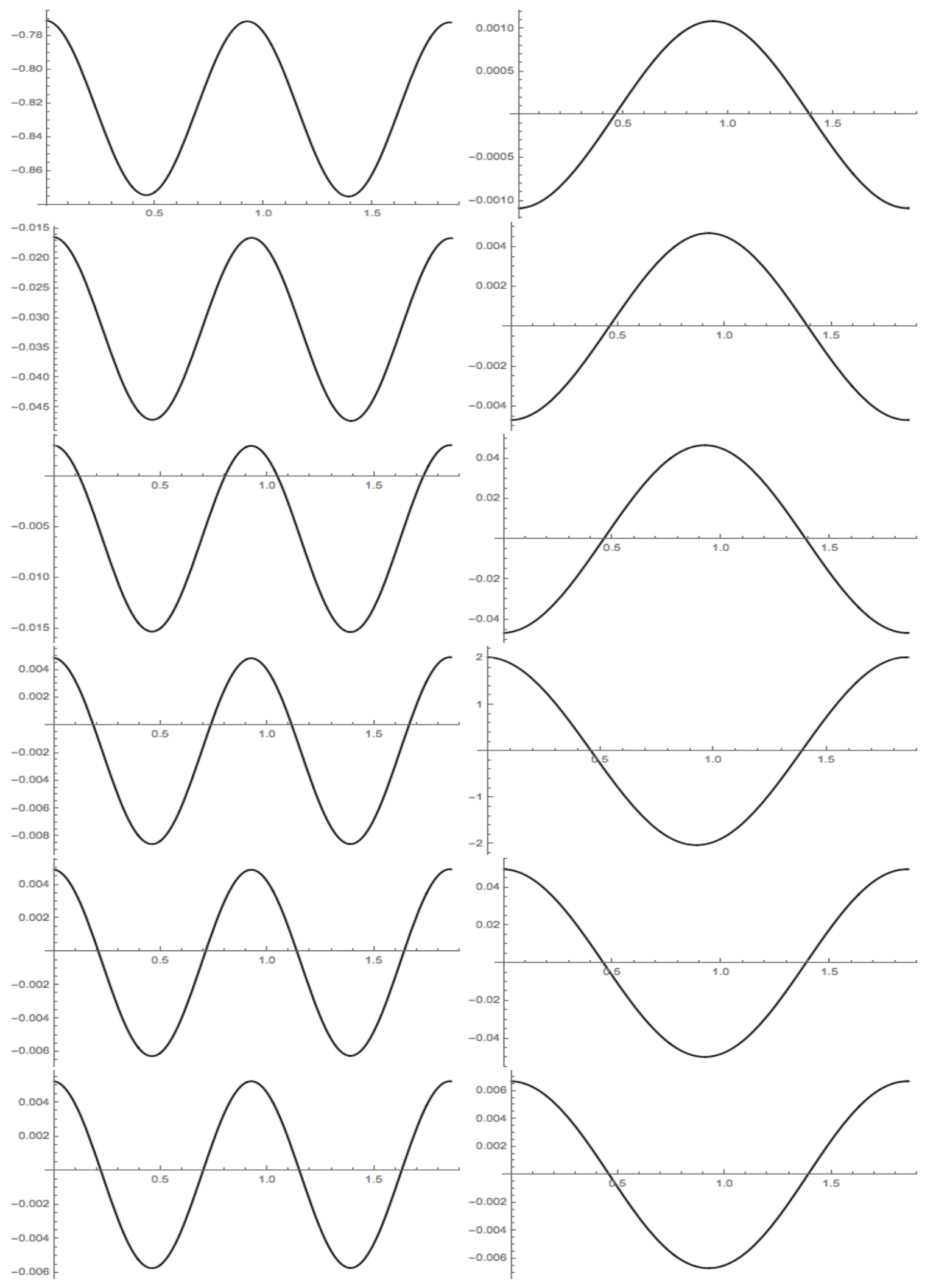

Figure 4: Time evolution of the Fourier components 1 to 12 of the solution on the 8th branch for $T=1.86$ (critical threshold of instability). 


\section{Torsional stability of longitudinal modes}

\subsection{Theoretical framework}

The purpose of this section is to insert the stability analysis of suspension bridges within a suitable theoretical framework. We explain why, for small longitudinal oscillations, one expects the system (6)-(7) to remain torsionally stable, and why, for large longitudinal oscillations, the system becomes unstable. Most of the tools introduced in this section are applications to our context of classical tools from the theory of ODE's that can be found e.g. in [14, 43.

We consider a (time) periodic solution $y$ of (9) and we denote by $T_{y}$ its period. Then we view the equation (7) for $\theta$ as an independent equation, that is, a linear hyperbolic equation of the form

$$
\alpha(x) \theta_{t t}=\left(a(x, t) \theta_{x}\right)_{x}+\beta(x)\left[\int_{0}^{L} b(x, t) \theta(x, t) d x\right]+c(x, t)\left[\int_{0}^{L} \gamma(x) \theta(x, t) d x\right]
$$

for suitable coefficients $a, b, c, \alpha, \beta, \gamma$; note that the coefficients $a, b, c$ depend on the periodic solution $y=y(x, t)$ of $(9)$.

Because of energy conservation, if the energy of the system is at some time concentrated on low Fourier coefficients of the solution, e.g. if the initial condition is smooth, then it remains so for all times. Hence, it seems sensible to simplify the problem by studying its projection onto the space spanned by $\left\{\sin \left(\frac{\pi}{L} x\right), \ldots, \sin \left(\frac{\nu \pi}{L} x\right)\right\}$, for some integer $\nu \geq 2$. This integer $\nu$ needs not (so che a volte si usa, ma non è corretto. "does not need" è meglio) to be the same as the integer $n$ in Section 3 . in order to state and prove a sufficient condition for stability we simply take $\nu=2$ whereas for our numerical experiments we take $\nu=n$.

For each $k=1, \ldots, \nu$ we multiply $\sqrt{19}$ by $\sin \left(\frac{k \pi}{L} x\right)$ and we integrate over $(0, L)$ : we obtain $\nu$ ODEs in the form

$$
\ddot{\theta}_{k}(t)+\sum_{j=1}^{\nu} \chi_{j k}(t) \theta_{j}(t)=0, \quad(k=1, \ldots, \nu)
$$

for some coefficients $\chi_{i j}$ depending only on $t$. We take the solution of $(20)$, that is,

$$
\theta(x, t)=\sum_{j=1}^{\nu} \theta_{j}(t) \sin \left(\frac{j \pi}{L} x\right)
$$

as an approximate solution of $(19)$. Since the time-dependent coefficients in 19 are computed in terms of the periodic solution $y$ of $(9)$, we have that

$$
\text { the functions } \quad t \mapsto \chi_{j k}(t) \text { are } T_{y} \text {-periodic } \forall j, k=1, \ldots, \nu \text {. }
$$

Moreover, these coefficients are symmetric: $\chi_{i j}(t) \equiv \chi_{j i}(t)$ for all $i, j$. We define the symmetric $\nu \times \nu$ matrix

$$
\boldsymbol{\Xi}(t)=\left(\chi_{j k}(t)\right)_{j, k=1, \ldots, \nu},
$$

which is $T_{y}$-periodic by $(22)$. Then we rewrite 20 in the vectorial form

$$
\ddot{W}+\boldsymbol{\Xi}(t) W=0,
$$

where $W(t)=\left(\theta_{1}(t), \ldots, \theta_{\nu}(t)\right)$ is the finite dimensional approximate vector containing the torsional components of the motion. Following [43, p.155, vol.1] we give the

Definition 2. We say that the system (23) is strongly stable if all its solutions are bounded in $\mathbb{R}$ and this property is preserved under small perturbations of $\boldsymbol{\Xi}(t)$. 
In turn, since the system (23) has coefficients which depend on the particular nonlinear longitudinal mode used to build the symmetric matrix $\Xi(t)$, we may characterize the stability of the modes as follows.

Definition 3. We say that a nonlinear longitudinal mode of (18) (see Definition 1) is torsionally stable if the system (23) is strongly stable; otherwise, we say that it is torsionally unstable.

Clearly, the boundedness of all solutions of the linear system (23) is equivalent to the stability of the trivial solution $W(t) \equiv 0$ and, therefore, to the torsional stability of the nonlinear longitudinal mode. Our purpose is precisely to study the stability of (23). In Section 4.2 we state and prove a sufficient condition for the stability in the case $\nu=2$ and we discuss how, for general systems such as (23), instability may arise. In Section 4.3 we compute numerically the thresholds of instability in dependence of the behavior of the nonlinear longitudinal mode which is used to built the coefficient matrix $\Xi$ in 23 .

\subsection{The case $\nu=2$}

A fairly useful tool to prove the stability for linear systems such as $(23)$ is the following criterion, see [43, Theorem II p.270, vol.1]:

Lemma 1. Assume that $a \nu \times \nu$ matrix $\mathbf{P}(t)$ is continuous and T-periodic for some $T>0$ and write $\mathbf{P}(t)=\mathbf{D}(t)+\mathbf{R}(t)$ where $\mathbf{D}(t)$ is the diagonal matrix containing the same elements as $\mathbf{P}(t)$ and $\mathbf{R}(t)=\mathbf{P}(t)-\mathbf{D}(t)$ is the "residual matrix" containing all the coefficients off the diagonal of $\mathbf{P}(t)$. Let $r_{1}$ and $r_{2}$ be two continuous T-periodic functions such that

$$
r_{1}(t) \mathbf{I} \leq \mathbf{R}(t) \leq r_{2}(t) \mathbf{I} \quad \forall t \in[0, T] .
$$

Let $\delta_{k}(t)(k=1, \ldots, \nu)$ be the elements of the diagonal matrix $\mathbf{D}(t)$. Assume that for all $s \in[0,1]$ the scalar Hill equations

$$
\ddot{z}_{k}+\left(\delta_{k}(t)+s r_{1}(t)+(1-s) r_{2}(t)\right) z_{k}(t)=0 \quad(k=1, \ldots, \nu)
$$

are strongly stable. Then the system $\ddot{Z}+\mathbf{P}(t) Z=0$ is strongly stable (here, $Z=Z(t) \in \mathbb{R}^{\nu}$ ).

In this section we study in detail the case $\nu=2$, since this case is simpler to handle and allows to prove a sufficient condition for the stability. Moreover, according to the detailed analysis of the TNB collapse by Smith-Vincent [41, p.21], the only torsional mode which developed under wind action on the bridge or on the model is that with a single node at the center of the main span, see also [18] for further evidence showing the importance of the second torsional component.

If $\nu=2$ then $(23)$ simply becomes

$$
\left(\begin{array}{l}
\ddot{\theta}_{1} \\
\ddot{\theta}_{2}
\end{array}\right)+\left(\begin{array}{cc}
\chi_{11}(t) & 0 \\
0 & \chi_{22}(t)
\end{array}\right)\left(\begin{array}{c}
\theta_{1} \\
\theta_{2}
\end{array}\right)+\left(\begin{array}{cc}
0 & \chi_{12}(t) \\
\chi_{12}(t) & 0
\end{array}\right)\left(\begin{array}{l}
\theta_{1} \\
\theta_{2}
\end{array}\right)=\left(\begin{array}{l}
0 \\
0
\end{array}\right)
$$

where we have emphasized the diagonal matrix $\mathbf{D}(t)$ and the residual matrix $\mathbf{R}(t)$. We point out that a simple and elegant form such as 250 is made possible by the fact that $\nu=2$ : the eigenvalues of the residual (symmetric) matrix $\mathbf{R}(t)$ are $\pm \chi_{12}(t)$ which means that

$$
-\left|\chi_{12}(t)\right| \mathbf{I} \leq \mathbf{R}(t) \leq\left|\chi_{12}(t)\right| \mathbf{I} \quad \forall t \in\left[0, T_{y}\right] .
$$

By (26), in the situation under study the $\nu=2$ families of Hill equations in (24) read

$$
\begin{aligned}
& \ddot{\theta}_{1}+\left(\chi_{11}(t)+\alpha \chi_{12}(t)\right) \theta_{1}=0 \\
& \ddot{\theta}_{2}+\left(\chi_{22}(t)+\alpha \chi_{12}(t)\right) \theta_{2}=0
\end{aligned} \quad(-1 \leq \alpha \leq 1) .
$$

Let us derive (27) in the limit case where $y=0$. 
Lemma 2. There exist $\gamma_{1}, \gamma_{2}>0$ such that, if $y \equiv 0$, then the function

$$
\theta(x, t)=\theta_{1}(t) \sin \left(\frac{\pi}{L} x\right)+\theta_{2}(t) \sin \left(\frac{2 \pi}{L} x\right)
$$

satisfies the projection of (7) onto $\operatorname{span}\left\{\sin \left(\frac{\pi}{L} x\right), \sin \left(\frac{2 \pi}{L} x\right)\right\}$ with $\theta_{1}$ and $\theta_{2}$ solving

$$
\ddot{\theta}_{1}+\gamma_{1} \theta_{1}=0, \quad \ddot{\theta}_{2}+\gamma_{2} \theta_{2}=0 \text {. }
$$

Proof. If $y \equiv 0$, then (7) reads

$$
\left(\frac{M}{3}+2 m \xi\right) \ell^{2} \theta_{t t}=G K \theta_{x x}+2 \ell^{2}\left\{H_{0}\left(\frac{\theta_{x}}{\xi^{2}}\right)_{x}-\frac{A E}{L_{c}}\left[\int_{0}^{L} \frac{s^{\prime \prime} \theta}{\xi^{3}}\right] \frac{s^{\prime \prime}}{\xi^{3}}\right\}
$$

and all the coefficients are now independent of $t$. The functions involved in this equation are either symmetric or skew-symmetric with respect to $x=\frac{L}{2}$ :

$$
s^{\prime \prime}(x), \xi(x), \sin \left(\frac{\pi}{L} x\right), \cos \left(\frac{2 \pi}{L} x\right) \text { are symmetric, } \quad s^{\prime}(x), \sin \left(\frac{2 \pi}{L} x\right), \cos \left(\frac{\pi}{L} x\right) \text { are skew-symmetric. }
$$

Take $\theta$ in the form $(28)$ and plug it into $(30)$; then multiply $(30)$ by $\sin \left(\frac{\pi}{L} x\right)$ and integrate over $(0, L)$. Several terms cancel due to (31): if an odd number of skew-symmetric functions is involved then the integral over $(0, L)$ vanishes. We end up with the first equation in 29 with

$$
\gamma_{1}=\frac{1}{A}\left(\frac{G K \pi^{2}}{2 L}+\frac{2 \pi^{2} \ell^{2} H_{0}}{L^{2}} \int_{0}^{L} \frac{\cos ^{2}\left(\frac{\pi}{L} x\right)}{\xi(x)^{2}} d x+\frac{2 A E \ell^{2}}{L_{c}}\left[\int_{0}^{L} \frac{s^{\prime \prime}(x) \sin \left(\frac{\pi}{L} x\right)}{\xi(x)^{3}} d x\right]^{2}\right)>0
$$

and

$$
A=\ell^{2} \int_{0}^{L}\left(\frac{M}{3}+2 m \xi(x)\right) \sin ^{2}\left(\frac{\pi}{L} x\right) d x .
$$

Then multiply $(30)$ by $\sin \left(\frac{2 \pi}{L} x\right)$ and integrate over $(0, L)$. By using again $(31)$, we find the second equation in 29 with

$$
\gamma_{2}=\frac{1}{B}\left(\frac{2 G K \pi^{2}}{L}+\frac{8 \pi^{2} \ell^{2} H_{0}}{L^{2}} \int_{0}^{L} \frac{\cos ^{2}\left(\frac{2 \pi}{L} x\right)}{\xi(x)^{2}} d x\right)>0, \quad B=\ell^{2} \int_{0}^{L}\left(\frac{M}{3}+2 m \xi(x)\right) \sin ^{2}\left(\frac{2 \pi}{L} x\right) d x .
$$

The proof is complete.

Needless to say, the equations (29) admit periodic (bounded!) solutions $\theta_{1}$ and $\theta_{2}$ for any initial data. We denote by $\mathbb{N}$ the set of nonnegative integers and we assume that

$$
\frac{T_{0}}{\pi} \sqrt{\gamma_{k}} \notin \mathbb{N} \quad \text { for } k=1,2
$$

where $T_{0}=\lim _{\|y\|_{\infty} \rightarrow 0} T_{y}$ is the period of the corresponding periodic solution of the linear problem. Clearly, 32 has probability 1 to occur among all possible choices of $\left(G, K, M, T_{0}, L, \ell\right) \in \mathbb{R}_{+}^{6}$.

Let $T_{y}$ be as in (22); if $(32)$ holds, we know that for $k=1,2$ there exists $n_{k} \in \mathbb{N}$ such that

$$
\frac{n_{k}^{2} \pi^{2}}{T_{0}^{2}}<\gamma_{k}<\frac{\left(n_{k}+1\right)^{2} \pi^{2}}{T_{0}^{2}}
$$

If $y \neq 0$ and $y, y_{x}, y_{x x} \rightarrow 0$ uniformly with respect to $t$, then

$$
\chi_{k k}(t) \rightarrow \gamma_{k} \quad(k=1,2), \quad \chi_{12}(t) \rightarrow 0 \quad \text { uniformly }
$$


and the system (27) converges to $(29)$, see Lemma 2. Therefore, by (33) and by continuity, we infer that there exists $\varepsilon>0$ such that if $\|y\|_{\infty}<\varepsilon$ then

$$
\frac{n_{k}^{2} \pi^{2}}{T_{y}^{2}} \leq \chi_{k k}(t)-\left|\chi_{12}(t)\right| \leq \chi_{k k}(t)+\left|\chi_{12}(t)\right| \leq \frac{\left(n_{k}+1\right)^{2} \pi^{2}}{T_{y}^{2}}
$$

for $k=1,2$ and for all $t \geq 0$. Then a stability criterion for the Hill equation due to Zhukovskii [44], see also [43, Test 1 p.697, vol.2], states that the two equations in (27) are strongly stable. In turn, Lemma 1 implies that the system $(25)$ is strongly stable. We have so proved the following statement.

Theorem 1. Assume (32) and let $y$ be a nonlinear longitudinal mode of (18). There exists $\varepsilon>0$ such that if $\|y\|_{\infty}<\varepsilon$, then the projection (23) of (7) onto $\operatorname{span}\left\{\sin \left(\frac{\pi}{L} x\right), \sin \left(\frac{2 \pi}{L} x\right)\right\}$ is strongly stable; therefore, $y$ is torsionally stable.

Clearly, a solution $y$ of 18 with small $L^{\infty}$-norm also has small energy. Theorem 1 says nothing about the stability of (23) when the periodic solution $y$ of $(9)$ has large amplitude (or energy). We now address this question.

Instead of (23) we consider a first order linear differential system such as

$$
\dot{Z}=A(t) Z
$$

where $Z \in \mathbb{R}^{2 \nu}$ for some $\nu \geq 2$ and $A$ is a $T$-periodic $2 \nu \times 2 \nu$ matrix for some $T>0$; the system (23) may be written in the form (34) by setting $Z=(W, \dot{W})$. Define a transfer map $Z(t) \mapsto Z(t+T)$ in terms of a suitable transition matrix $M$, that is, $Z(t+T)=M Z(t)$ with $Z$ being a solution of (34). It is clear that if all the (complex) eigenvalues of $M$ have modulus 1 , then the solutions of (34) are globally bounded and therefore the trivial solution $Z \equiv 0$ of $(34)$ is stable. The numerical procedure described in Section 4.3 computes these eigenvalues.

Ortega [34] considers a family of nonlinear Hill equations such as

$$
\ddot{z}+a(t) z+f(z)=0 \quad \text { with } a(t+T)=a(t) ;
$$

a typical example is $f(z)=z^{3}$ but many other nonlinearities $f$ yield the same behavior. Taking $Z:=(z, \dot{z})$, this equation may be reduced to a first order nonlinear $2 \times 2$ system of the kind of (34):

$$
\dot{Z}=A(t) Z+F(Z) .
$$

Under suitable conditions on the nonlinearity $F$, Ortega proves that if the trivial solution of (34) is stable, then also the trivial solution of (36) is stable. This sufficient condition shows that a linearization process is legitimate, if one aims to study the stability of (36).

In their paper, Cazenave-Weissler [13] analyzed the stability of simple modes of (12) by using techniques from the Floquet theory, combined with Poincaré maps and the Hill equations. They were able to prove that, if the energy is sufficiently large, then simple modes of $(12)$ become unstable: they take advantage of the fact that $(12)$ is "almost linear".

For a different nonlinear and local system of ODE's it was proved in [9] that the same fact occurs, large energies yield instability of modes. The analysis in [9] is fairly precise and is made possible by the fact that the linearized dynamical system leads to some Mathieu equations (a particular case of the Hill equations) for which more precise theoretical tools are available.

Therefore, both for nonlinear nonlocal problems [13] and for nonlinear local problems [9], the instability of the nonlinear longitudinal modes of (18) with large energy has to be expected. The system (6)-(7) is certainly much more complicated than the systems considered there, and a rigorous proof of the torsional instability at high energies seems out of reach, but we believe that it is governed by the same phenomena. In the next section we will see that the numerical experiments support our belief. 


\subsection{Thresholds of torsional instability}

Take a $k$-th nonlinear longitudinal mode $y$ of (18) having period $T_{y}$ (see Definition 1 ) and use it to build the coefficient matrix $\Xi(t)$ in 23 . Then transform 23 into 34 by setting $Z=(W, \dot{W})$. We choose $2 \nu$ different initial conditions $Z(0)=e_{k}$, where now $\left\{e_{k}\right\}_{k=1, \ldots, 2 \nu}$ is the canonical basis in $\mathbb{R}^{2 \nu}$, and for each initial condition we solve $[34)$ in $\left[0, T_{y}\right]$. Let $M$ be the transition matrix obtained by placing into adjacent columns the solutions $Z\left(T_{y}\right)$ of (34) for all the initial conditions $Z(0)=e_{k}$ $(k=1, \ldots, 2 \nu)$. Compute its eigenvalues and consider the largest in modulus: when such value is larger than 1 the longitudinal mode is torsionally unstable.

In this section we implement the just described procedure and we describe the numerical computations of the thresholds of torsional instability for each branch of periodic longitudinal modes. Several different indicators are used to measure the instability. The values of the parameters involved in the equations are taken as for the collapsed TNB, see Section 4.4 .

Table 2 summarises the thresholds of instability for the different branches of longitudinal modes: the second column is the energy threshold, the third column is the period of the longitudinal mode at the threshold, and the fourth is a measure of the amplitude of oscillation, that is, the gap between the maximum and the minimum of the longitudinal mode $y$ :

$$
\Delta:=\max _{x, t} y(x, t)-\min _{x, t} y(x, t) .
$$

\begin{tabular}{|c|c|c|c|}
\hline Branch & Energy $(M J)$ & Period $(s)$ & $\Delta(m)$ \\
\hline 1 & 38. & 11.22 & 5.8 \\
2 & 51.8 & 8.46 & 10 \\
3 & 15.5 & 5.48 & 6.5 \\
4 & 53.7 & 3.97 & 7.8 \\
5 & 74.1 & 3.14 & 6.9 \\
6 & 56.6 & 2.53 & 4.5 \\
7 & 91.4 & 2.18 & 5.2 \\
8 & 95.8 & 1.86 & 4.6 \\
9 & 87.1 & 1.59 & 3.8 \\
10 & 82.1 & 1.38 & 3.3 \\
\hline
\end{tabular}

Table 2: Thresholds of instability on each branch of nonlinear modes.

A careful look at the video [42] and the data in the Report [2] confirm that the oscillations prior to the TNB collapse were of the order of a few meters, as in our numerical results, see the values of $\Delta$ in Table 2, The period at the threshold of instability should be compared with the period of the longitudinal mode on the same branch when the energy is close to 0 , that is, when the nonlinear system 18 is close to linear, see Table 1 .

Let us now introduce an effective tool to measure the torsional instability which allows the comparison of different longitudinal modes. The Floquet Theorem (see [14, Theorem 8.23]) states that, if $\Psi(t)$ is a fundamental matrix solution of (34), then there exists a matrix $B$ and a $T_{y}$-periodic matrix $P$ such that $\Psi(t)=P(t) e^{t B}$; note that both $B$ and $P$ may be complex-valued. The eigenvalues $\lambda_{1}, \ldots, \lambda_{2 \nu}$ of $e^{T_{y} B}$ are called the characteristic multipliers of (34): if $V_{1}, \ldots, V_{2 \nu}$ denote the corresponding normalized eigenvectors, then the solution $Z_{j}$ of (34) satisfying the initial condition $Z_{j}(0)=V_{j}$ (for some $j=1, \ldots, 2 \nu)$ also satisfies $Z_{j}\left(T_{y}\right)=\lambda_{j} Z_{j}(0)$ and, in turn, $Z_{j}\left(m T_{y}\right)=\lambda_{j}^{m} Z_{j}(0)$ for any integer 
$m \geq 1$. Whence $\left|\lambda_{j}\right|^{1 / T_{y}}$ represents the rate of growth of the amplitude of oscillation of $Z_{j}$. This leads to the following definition:

Definition 4. We call expansion rate of (34) the largest rate of growth for solutions of (34):

$$
\mathcal{E R}:=\max _{j}\left|\lambda_{j}\right|^{1 / T_{y}} .
$$

For every branch of nonlinear longitudinal modes we compute the expansion rate of (34), that is, the expansion rate of the torsional component of the motion. Figure 5 displays the expansion rate
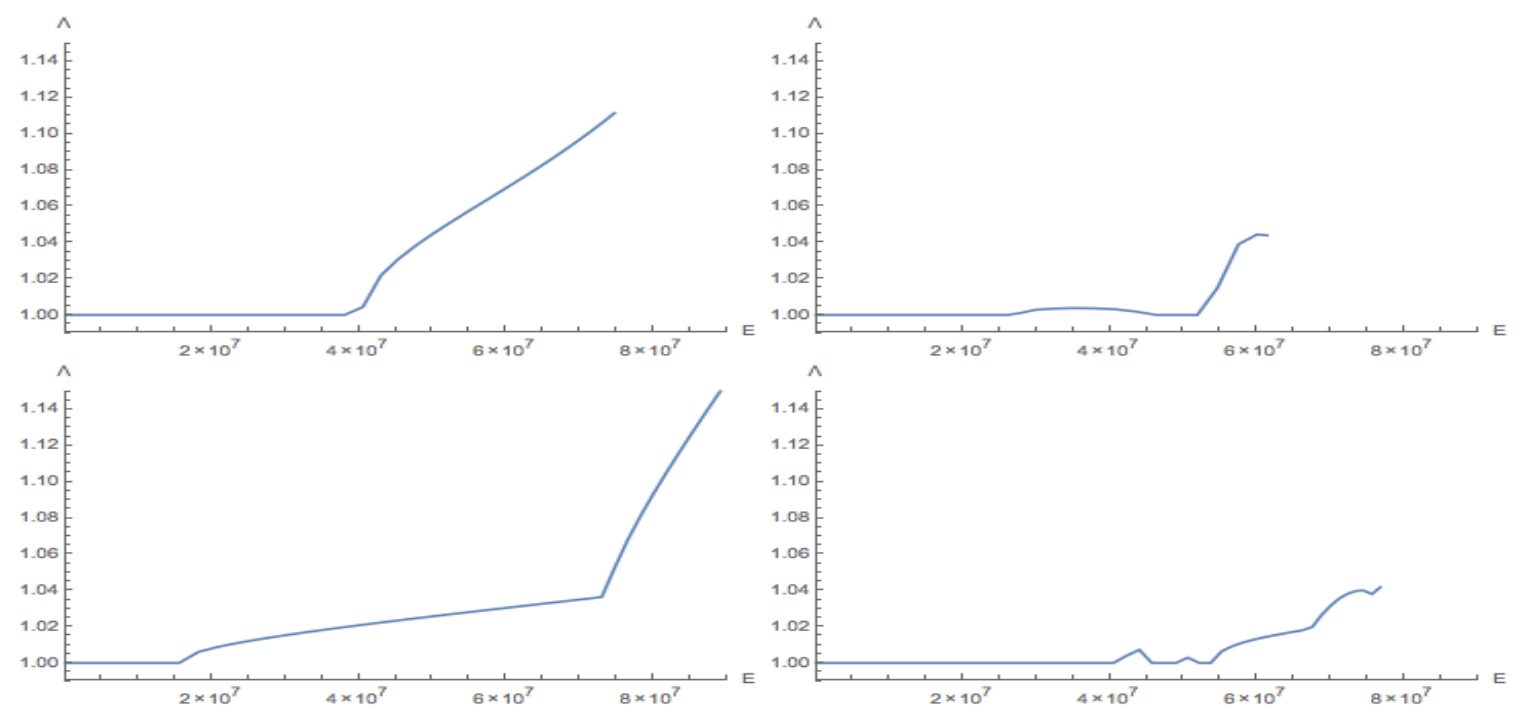

Figure 5: Expansion rate for modes 1 to 4 with respect to the energy.

as a function of the energy for the branches of nonlinear longitudinal modes of order 1 to 4 whereas Figure 6 displays the same for the modes of order 5 to 10 . Note that the vertical axis (the $\mathcal{E} \mathcal{R}$-axis) has different scales in the two figures. The reason is that $\mathcal{E} \mathcal{R}$ is much larger for high nonlinear longitudinal modes. All the plots clearly show that there exists a threshold above which the torsional stability of the longitudinal mode is lost, that is, where the expansion rate exceeds 1 .

Table 3 summarises the expansion rates on all the branches that we considered, for different values of the energy. Some values in Table 3 are not available because the Newton algorithm would require too small increments of $T$ in order to follow the branch of solutions. Note that the energy of a longitudinal mode is related to the amplitude of oscillation $\Delta$ and, for a given energy, higher modes have smaller amplitudes. Therefore, for the same amplitude of oscillation, the expansion rate of the 9th and 10th longitudinal mode is larger than that of the 5 th, see also Table 2 . 

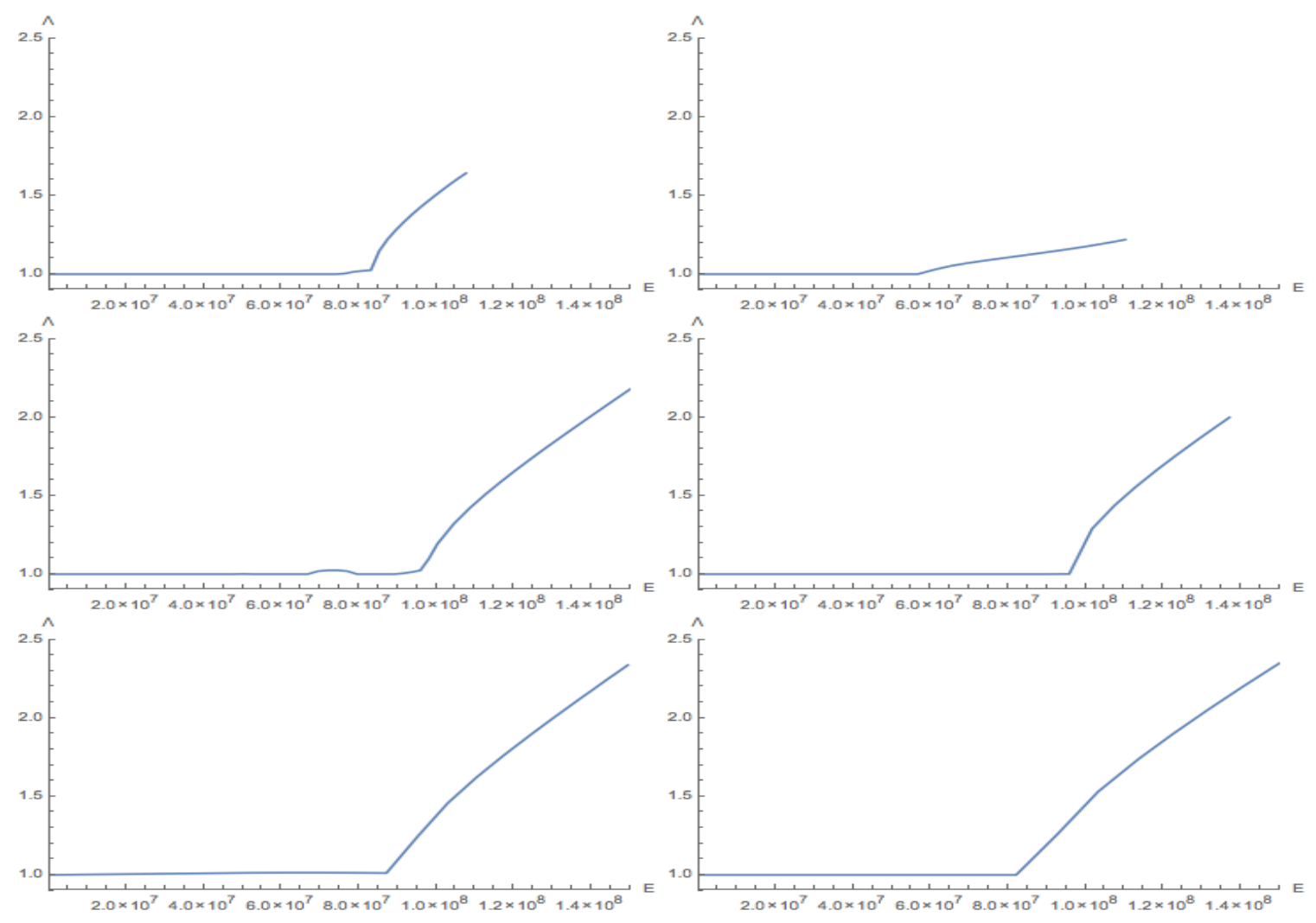

Figure 6: Expansion rate for modes 5 to 10 with respect to the energy.

\begin{tabular}{|c|c|c|c|c|c|c|c|}
\hline Branch & 2 & 4 & 6 & 8 & 10 & 12 & 14 \\
\hline 1 & 1. & 1. & 1.0662 & - & - & - & - \\
2 & 1. & 1.00365 & 1.03904 & - & - & - & - \\
3 & 1.00614 & 1.02071 & 1.02961 & 1.08141 & 1.20949 & - & - \\
4 & 1. & 1. & 1.01287 & - & - & - & - \\
5 & 1. & 1. & 1.00001 & 1.01521 & 1.50051 & - & - \\
6 & 1. & 1. & 1. & 1.09919 & 1.16332 & - & - \\
7 & 1. & 1. & 1. & 1. & 1.09852 & 1.58567 & 1.97158 \\
8 & 1. & 1. & 1. & 1. & 1.00112 & 1.66552 & - \\
9 & 1. & 1. & 1.01322 & 1.01353 & 1.24852 & 1.76429 & 2.12488 \\
10 & 1. & 1. & 1. & 1. & 1.25447 & 1.73715 & 2.05263 \\
\hline
\end{tabular}

Table 3: Expansion rates versus energy (in MJ). 
According to Eldridge [2, V-3], a witness on the day of the TNB collapse, the bridge appeared to be behaving in the customary manner and the motions were considerably less than had occurred many times before. From [2, p.20] we also learn that in the months prior to the collapse one principal mode of oscillation prevailed and that the modes of oscillation frequently changed. On the day of the collapse, the torsional oscillations at the TNB started suddenly and, according to Farquharson [2, V-10], the motions, which a moment before had involved a number of waves (nine or ten) had shifted almost instantly to two. These observations show that the transfer of energy (from longitudinal to torsional) depends on the particular excited mode.

Table 3 explains why torsional oscillations did not appear earlier at the TNB even in presence of larger longitudinal oscillations, as reported by Eldridge: there are longitudinal modes which have a very small expansion rate. Moreover, Table 3 also explains why torsional oscillations appeared with smaller longitudinal oscillations: the 9 th and 10th longitudinal mode appear more prone to generate torsional oscillations because they have large expansion rate $\mathcal{E} \mathcal{R}$.

\subsection{Glossary and constants}

In this section we list the constants considered throughout the paper.

- $y=y(x, t)$ downwards vertical displacement of the center of the deck

- $\theta=\theta(x, t)$ torsional angle of the deck

- $\ell$ half width of the deck $(6 m)$

- $L$ length of the deck $(853.44 m)$

- $M$ mass linear density of the deck $(7198 \mathrm{~kg} / \mathrm{m})$

- E Young modulus of the deck and of the cables (210GPa)

- I linear density of the moment of inertia of the cross section $\left(0.15 \mathrm{~m}^{4}\right)$

- $G$ shear modulus of the deck (81GPa)

- $K$ torsional constant of the deck $\left(6.44 \cdot 10^{-6} m^{4}\right)$

- $m$ mass linear density of the cable $(981 \mathrm{~kg} / \mathrm{m})$

- $H_{0}$ horizontal component of the tension of the cables $(58300 k N)$

- $H(x)$ tangential component of the tension of the cables

- $L_{c}$ length of the cable $(868.62 m)$

- $s_{0}$ length of the longest hanger $(72 m)$

- $A$ area of the section of the cable $\left(0.1228 m^{2}\right)$

- Bridge at rest means bridge fully mounted, operational at equilibrium.

- Bridge unloaded means that the deck is not mounted. 


\section{Conclusions}

For long time the aerodynamic effects have been considered the only cause of the TNB collapse. But, as quickly surveyed in the Introduction, no purely aerodynamic explanation is nowadays able to justify the origin of the torsional oscillation, which is the main culprit for the collapse. In this paper we suggest a new nonlinear model, which improves [5], and we focus our attention on the nonlinear structural behavior of suspension bridges. To this end, we follow a suggestion by Irvine [23] and we isolate the bridge from aerodynamic effects and dissipation. The new model and the resulting evolution PDEs which describe the dynamics of suspension bridges are presented in Section 2 .

In Section 3 we explain how to find periodic longitudinal oscillations as solutions of a nonlinear system of ODEs: contrary to linear systems, the period depends on the energy and on the amplitude of the oscillation.

In Section 4.1 we describe the theoretical framework governing the structural instability of suspension bridges. Our analysis is based on tools from the Floquet theory and a sufficient condition for the stability at low energy is obtained in Section 4.2. Then, in Section 4.3, we put into the model the quantitative parameters of the collapsed TNB and we compute its thresholds of instability. The results are in line with the values observed on the day of the collapse and well fit the Report [2]. First, the computed critical amplitude of longitudinal oscillations is the same as in the video [42. Second, our results explain why the TNB withstood larger longitudinal oscillations on low modes, but failed for smaller longitudinal oscillations on higher modes, see [2, pp.28-31]. Our results show that the origin of torsional instability is structural.

It is clear that, in absence of wind or external loads, the deck of a bridge remains still. On the other hand, when the wind hits a bluff body (such as the deck of the TNB) the flow is modified and goes around the body. Behind the deck, or a "hidden part" of it, the flow creates vortices which are, in general, asymmetric. This asymmetry generates a forcing lift which starts the vertical oscillations of the deck. Up to some minor details, this explanation is shared by the whole community and it has been studied with great precision in wind tunnel tests, see e.g. [27, 39, 40]. The vortices induced by the wind increase the internal energy of the structure and generate wide longitudinal oscillations which look periodic in time. This is the point where our analysis starts, that is when the longitudinal oscillations of the structure reach a periodic motion which is maintained in amplitude by a somehow perfect equilibrium between the input of energy from the wind and internal dissipation. Throughout this paper we have seen that, in such situation, if the longitudinal oscillation is sufficiently large, then a structural instability appears: this is the onset of torsional oscillations. In order to obtain a more accurate description of the dynamics of suspension bridges, the next step should be to take into account also the behavior of the aerodynamic and dissipation effects after the appearance of the torsional oscillations.

Acknowledgements. The Authors are partially supported by the PRIN project Equazioni alle derivate parziali di tipo ellittico e parabolico: aspetti geometrici, disuguaglianze collegate, e applicazioni, and they are members of the Gruppo Nazionale per l'Analisi Matematica, la Probabilità e le loro Applicazioni (GNAMPA) of the Istituto Nazionale di Alta Matematica (INdAM).

\section{References}

[1] B. Akesson, Understanding bridges collapses, CRC Press, Taylor \& Francis Group, London (2008)

[2] O.H. Ammann, T. von Kármán, G.B. Woodruff, The failure of the Tacoma Narrows Bridge, Federal Works Agency, Washington D.C. (1941)

[3] A. Arena, W. Lacarbonara, Nonlinear parametric modeling of suspension bridges under aeroelastic forces: torsional divergence and flutter, Nonlinear Dynamics 304, 72-90 (2012) 
[4] G. Arioli, F. Gazzola, A new mathematical explanation of what triggered the catastrophic torsional mode of the Tacoma Narrows Bridge collapse, Appl. Math. Modelling 39, 901-912 (2015)

[5] G. Arioli, F. Gazzola, On a nonlinear nonlocal hyperbolic system modeling suspension bridges, Milan J. Math. DOI 10.1007/s00032-015-0239-9

[6] G. Bartoli, P. Spinelli, The stochastic differential calculus for the determination of structural response under wind, J. Wind Engineering and Industrial Aerodynamics 48, 175-188 (1993)

[7] E. Berchio, A. Ferrero, F. Gazzola, Structural instability of nonlinear plates modelling suspension bridges: mathematical answers to some long-standing questions, preprint (2015)

[8] E. Berchio, F. Gazzola, A qualitative explanation of the origin of torsional instability in suspension bridges, Nonlin. Anal. T.M.A. 121, 54-72 (2015)

[9] E. Berchio, F. Gazzola, C. Zanini, Which residual mode captures the energy of the dominating mode in second order Hamiltonian systems?, (arXiv:1410.2374)

[10] K.Y. Billah, R.H. Scanlan, Resonance, Tacoma Narrows Bridge failure, and undergraduate physics textbooks, Amer. J. Physics 59, 118-124 (1991)

[11] F. Bleich, Dynamic instability of truss-stiffened suspension bridges under wind action, Proceedings ASCE 74, 1269-1314 (1948)

[12] E.I. Butikov, Subharmonic resonances of the parametrically driven pendulum, J. Phys. A: Math. Gen. 35, 6209-6231 (2002)

[13] T. Cazenave, F.W. Weissler, Unstable simple modes of the nonlinear string, Quart. Appl. Math. 54, 287-305 (1996)

[14] C. Chicone, Ordinary differential equations with applications, (2nd Edition) Springer (2006)

[15] N.J. Delatte, Beyond failure, ASCE Press (2009)

[16] R.W. Dickey, Stability of periodic solutions of the nonlinear string, Quart. Appl. Math. 38, 253-259 (1980)

[17] G. Gentile, V. Mastropietro, M. Procesi, Periodic solutions for completely resonant nonlinear wave euations with Dirichlet boundary conditions, Commun. Math. Phys. 256, 437-490 (2005)

[18] F. Gazzola, Mathematical models for suspension bridges, MS\&A Vol. 15, Springer (2015)

[19] M. Ghisi, M. Gobbino, Stability of simple modes of the Kirchhoff equation, Nonlinearity 14, 1197-1220 (2001)

[20] D. Green, W.G. Unruh, Tacoma Bridge failure - a physical model, Amer. J. Physics 74, 706-716 (2006)

[21] G. Herrmann, W. Hauger, On the interrelation of divergence, flutter and auto-parametric resonance, Ingenieur-Archiv 42, 81-88 (1973)

[22] D. Imhof, Risk assessment of existing bridge structure, PhD Dissertation, University of Cambridge (2004) See also http://www.bridgeforum.org/dir/collapse/type/ for the update of the Bridge failure database

[23] H.M. Irvine, Cable structures, MIT Press Series in Structural Mechanics, Massachusetts (1981)

[24] A. Jenkins, Self-oscillation, Physics Reports 525, 167-222 (2013)

[25] G. Kirchhoff, Vorlesungen über mathematische physik: mechanik, Section 29.7, Teubner, Leipzig (1876)

[26] W. Lacarbonara, Nonlinear structural mechanics, Springer (2013)

[27] A. Larsen, Aerodynamics of the Tacoma Narrows Bridge - 60 years later, Struct. Eng. Internat. 4, 243-248 (2000)

[28] C. Lee, Periodic solutions of beam equations with symmetry, Nonlin. Anal. T.M.A. 42, $631-650$ (2000) 
[29] J.Q. Liu, Free vibrations for an asymmetric beam equation, Nonlin. Anal. T.M.A. 51, 487-497 (2002)

[30] J.Q. Liu, Free vibrations for an asymmetric beam equation, II, Nonlin. Anal. T.M.A. 56, 415-432 (2004)

[31] J.L. Luco, J. Turmo, Effect of hanger flexibility on dynamic response of suspension bridges, J. Engineering Mechanics 136, 1444-1459 (2010)

[32] P.J. McKenna, Torsional oscillations in suspension bridges revisited: fixing an old approximation, Amer. Math. Monthly 106, 1-18 (1999)

[33] P.J. McKenna, Oscillations in suspension bridges, vertical and torsional, Disc. Cont. Dynam. Systems 7, 785-791 (2014)

[34] R. Ortega, The stability of the equilibrium of a nonlinear Hill's equation, SIAM J. Math. Anal. 25, 13931401 (1994)

[35] B.G. Pittel, V.A. Yakubovich, Application of the theory of parametric resonance to explain the collapse of the Tacoma Narrows Bridge (Russian), Uspekhi Mat. Nauk. 15, 183-184 (1961)

[36] B.G. Pittel, V.A. Yakubovich, A mathematical analysis of the stability of suspension bridges based on the example of the Tacoma Bridge (Russian), Vestnik Leningrad Univ. 24, 80-91 (1969)

[37] P.H. Rabinowitz, Free vibrations for a semilinear wave equation, Comm. Pure Appl. Math. 31, 31-68 (1978)

[38] R.H. Scanlan, The action of flexible bridges under wind, I: flutter theory, II: buffeting theory, J. Sound and Vibration 60, 187-199 \& 201-211 (1978)

[39] R.H. Scanlan, Developments in low-speed aeroelasticity in the civil engineering field, AIAA Journal 20, 839-844 (1982)

[40] R. Scott, In the wake of Tacoma. Suspension bridges and the quest for aerodynamic stability, ASCE Press (2001)

[41] F.C. Smith, G.S. Vincent, Aerodynamic stability of suspension bridges: with special reference to the Tacoma Narrows Bridge, Part II: Mathematical analysis, Investigation conducted by the Structural Research Laboratory, University of Washington Press, Seattle (1950)

[42] Tacoma Narrows Bridge collapse, http://www.youtube.com/watch?v=3mclp9QmCGs (1940)

[43] V.A. Yakubovich, V.M. Starzhinskii, Linear differential equations with periodic coefficients, J. Wiley \& Sons, New York (1975) (Russian original in Izdat. Nauka, Moscow, 1972)

[44] N.E. Zhukovskii, Finiteness conditions for integrals of the equation $d^{2} y / d x^{2}+p y=0$ (Russian), Mat. Sb. 16, 582-591 (1892) 